\title{
Properties of abundance gradient along the Galactic disk and the role of LAMOST
}

\author{
J.L. Hou ${ }^{1}$, L. Chen ${ }^{1}$, J.C. Yu ${ }^{1}$, J. Sellwood ${ }^{2}$ and C. Pryor ${ }^{2}$ \\ ${ }^{1}$ Shanghai Astronomical Observatory, CAS, Shanghai, 200030, China \\ email: houjl, chenli, yujc@shao.ac.cn \\ ${ }^{2}$ Department of Physics and Astronomy, Rutgers University, 136 Frelinghuysen Road, \\ Piscataway, NJ 08854, USA \\ email: sellwood@physics.rutgers.edu
}

\begin{abstract}
In this paper, we present our recent work on the evolution of abundance gradients along the Milky Way disk based on the Geneva Copenhagen Survey (GCS) and Radial Velocity Experiment (RAVE) data. We will also discuss the role of the LAMOST Milky Way disk survey in clarifying the properties of metallicity breaks observed through open clusters and young tracers along the Milky Way disk. It is believed that the Galactic disk forms inside-out, in which the stellar population at increasing radii is younger and more metal poor. This picture is consistent with most Galactic Chemical Evolution (GCE) models which also predict a tight correlation between the metallicity and age of stars at a given radius. However, it is only a result of "steady state" and no dynamical evolution effects were taken into account. We have selected two stellar samples from GCS and RAVE, each sample contains about 10,000 local thindisk, main-sequence stars. We use the guiding radius which is determined by the conservation of z-direction angular momentum, to eliminate the blurring effects. And also use the effective temperature of the main sequence stars as a proxy of stellar age. It is shown that the metallicity gradient flattens as the age increases. This is not consistent with our previous GCE prediction, but can be explained by radial mixing effects. In order to further demonstrate the abundance breaks observed in the Galactic disk we have proposed, and have been carrying out, an open cluster survey project based on LAMOST. We plan to observe at least 400 open clusters in the northern Galactic sky. From the observations, we will get uniform parameters for those clusters with radial velocity and metallicities. We anticipate that this uniform open cluster sample could clarify the observed abundance break around the Milky Way disk corotation radius and also give a more robust result concerning the evolution of the abundance gradient.
\end{abstract}

Keywords. Open Clusters, Abundance, Gradients, Surveys

\section{Introduction}

The disk is the defining stellar component of a disk galaxy since it contains almost all of the baryonic angular momentum. Understanding its formation is the most important goal of galaxy formation theory. There are two main clues which can trace the history of disk evolution. One comes from stellar kinematics from which we are able to learn disk heating history. The other is the abundances in the interstellar medium (ISM), stars and stellar moving groups or clusters. The abundances in stars retains detailed history of star formation and disk evolution. Especially, the radial abundance gradient along the disk is an essential ingredient in an accurate picture of galaxy formation and evolution. The gradient has close relations with various disk processes, such as gas infall time scale, gas inflow, stellar migration, and even dynamics of the Galactic bar and spiral arms. 
During the past twenty years, the existence of an abundance gradient along the Milky Way disk has been well established by observations using various tracers. A monotonically decreasing radial gradient can easily be explained by a simple GCE model assuming the infall time scale varied with radius smoothly (Hou, Prantzos \& Boissier 2000). However, two questions remain to be addressed both theoretically and observationally. One is the spatial distributions of the gas and stellar abundances along the disk. Is the linear behavior of the gradient universal for all tracers? Is the gradient break at the galactocentric distance of around $8-10 \mathrm{kpc}$, observed in the open clusters sample by Twarog, Ashman \& Anthony-Twarog(1997) and Lepine et al. (2011), real? The other question is how it evolves during the history of disk evolution. Is the disk abundance gradient steeper or flatter in the past? What is the main mechanism that drives the evolution of the abundance gradient?

In this contribution, we will first summarize the current observational status concerning the abundance gradients along the Milky Way disk, mainly the observed breaks from the open clusters sample and other tracers. Then we will introduce our recent work about the evolution of the abundance gradient using star samples from the Geneva-Copenhagen Sample (Nordstrom et al. 2004; Holmberg et al. 2009) and RAVE data (Steinmetz et al. 2006. In this, we try to test the radial migration theory by tracing the evolution of abundance gradients of stars with different ages. Finally, we will present our project using China's LAMOST telescope to observe a large sample of open clusters in the Galactic disk.

\section{Breaks of the abundance gradients along the Galactic disk}

The observed disk abundance gradient and its evolution provide the opportunity to test theories of disk chemical evolution and stellar nucleosynthesis. However, the shape of the gradient, that is the variation of the chemical abundance (either in ISM or stars and clusters) as a function of distance from the Galactic center is still not well fixed observationally.

During the past years, a linear oxygen or/and iron abundance gradients about of $-0.05 \sim-0.07 \mathrm{dex} \mathrm{kpc}^{-1}$ was obtained by observing young stars (B stars and Cepheids) and HII regions (Andrievsky et al. 2004; Rudolph et al. 2006; Pedicelli et al. 2009), Planetary Nebulae (Maciel et al. 2006) and Open Clusters (Friel 1999; Carraro et al. 1998; Chen et al. 2003; Magrini et al. 2009; Lepine et al. 2011). But this linear behaviour of the gradients has been questioned by Twarog, Ashman \& Anthony-Twarog (1997, hearafter TAA97). They found that the metallicity distribution of open clusters with regard to galactocentric distance can be best described by two distinct zones, with a sharp discontinuity at $R_{G C}=10 \mathrm{kpc}$. Between $R_{G C}=6.5 \mathrm{kpc}$ and $10 \mathrm{kpc}$, the clusters have a mean metallicity of 0.0 dex with, at best, weak evidence for a shallow gradient over this range, while those beyond $10 \mathrm{kpc}$ have a mean value of about -0.30 dex. TAA97 put forth an alternative description, the step function, about the radial abundances distribution of the open clusters. This two-step distribution seems quite similar to the nebula results of Simpson et al. (1995). However, a careful check with cluster ages shows that the sample inside $10 \mathrm{kpc}$ is heavily weighted towards clusters younger than $1 \mathrm{Gyr}$, while the outer clusters are predominantly older.

Recently, this break in the abundance gradient along the Milky Way disk was also shown based on high resolution data of open clusters and Cepheids by Lepine et al. (2011). They showed that the existence of a sharp step in the abundance gradients coincides with the Milky Way disk corotation radius. This implies that the discontinuity of the gradient may well be related to the spiral arms and galactic dynamic evolution. 
Based on 27 nearby face-on disk galaxies, Scarano et al. (2013) identified two classes of the discontinuities of the gradient for nearby disk spirals: smooth breaks and step breaks. The position at which inflexion takes place is well correlated with the corotation radius for all galaxy discs. This phenomena might be related to various mechanisms which need to be taken into account in the new updated chemical evolution models. The most important factor is the variation of gas density around corotation radius, which subsequently affects the Star Formation Rate (SFR) that is also induced by density waves in spiral galaxies (Wyse \& Silk 1989).

\section{Time evolution of the abundance gradients}

Another important question regarding the radial abundance gradient is how it changes with tracers of different ages during the history of the disk evolution. With the help of large sample of open clusters and planetary nebulae $(\mathrm{PNe})$, we are now able to explore this question observationally. The estimated ages of open clusters and planetary nebulae of various types span a large fraction of the age of the Galaxy. Observations of the abundances of those objects across the Milky Way disk have provided some important information on the past history of the abundance gradients. Indeed, current available data seems to show that the gradient is steeper in the past (Maciel et al. 2006; Chen, Hou \& Zhao 2008). This is also consistent with classical chemical evolution model (Hou et al. 2000; Fu et al. 2009).

The situation may not be so simple. In the classical model of GCE, the system is only open for gas (allowing for infall, outflow, and radial inflows). Stars are assumed to be remained where they were born. It is this assumption that makes us capable of reconstructing the history of Milky Way Galaxy according to the observed patterns, relations, such as abundance ratios, metallicity distribution function (MDF), age-metallicity relation (AMR), age-velocity relation(AVR), abundance gradient and so on. If a significant fraction of stars in the solar neighborhood are originated from inner disk or outer disk due to radial mixing process (Sellwood \& Binney 2002; Haywood 2008), there would be explicit effects on the abundance gradient along the disk. Also the local MDF would not be suitable to trace the local star formation history.

An additional consequence of radial mixing is that the radial metallicity gradient of a generation of stars is gradually flattened over time. This could be tested by well defined stellar samples which have full $6 \mathrm{D}$ phase space coordinates: distance, proper motion, radial velocity and sky position.

A radial velocity can be measured spectroscopically at any distance, but a reliable distance can be obtained only for nearby stars. Recently, Yu et al. (2012) assembled a sample of local, main-sequence stars having estimated effective temperatures, metallicities and full space motions. Based on this sample, Yu et al. (2012) find an apparent flattening of the metallicity gradient with decreasing effective temperature for RAVE stars and is more convincing in the better-quality data of the GCS sample.

An important result of $\mathrm{Yu}$ et al. (2012) is presented in Figure 1, we can see that the there is an apparent flattening of the metallicity gradient with decreasing $T_{\text {eff }}$ based on local star sample. Older stars seem to have a flatter gradient in the solar neighborhood, strong evidence showing that older stars could have undergone radial migration. This estimated gradient is in the range of other measurements from stars. For example, Coşkunoğlu et al. (2011) derived a metallicity gradient of $-0.051 \mathrm{dex} \mathrm{kpc}^{-1}$ for F-dwarfs, and they also find a shallower gradient for the slightly older G-dwarfs.

However, there are also opposite conclusions in terms of the observed evolution of abundance gradient, especially concerning the stellar tracers. Maciel et al. (2006) claim 


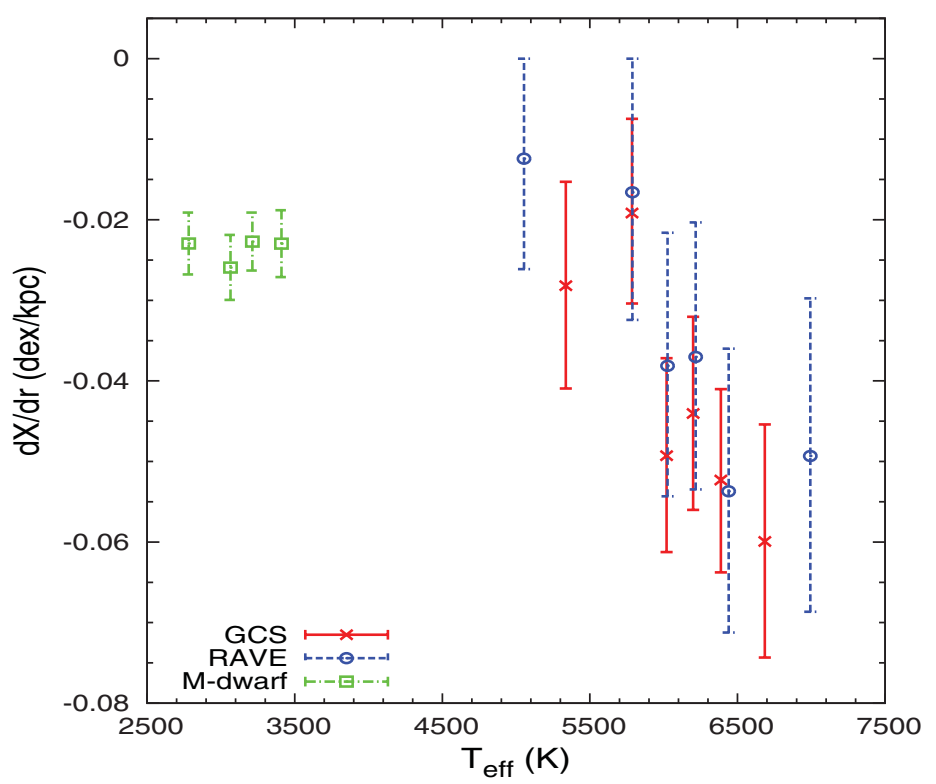

Figure 1. The metallicity gradient, $d[F e H] / d r$ estimated from the GCS sample (triangles), and from the RAVE sample (open circles). The error bars show the 95 Monte Carlo simulation with the adopted uncertainties(see $\mathrm{Yu}$ et al. 2012).

a steeper abundance gradient for older PNe, although their estimated gradients for the different elements show a large scatter and their ages are less reliable than those of star clusters. It should be noted that Stanghellini \& Haywood (2010) found that, for a sample of $\mathrm{PNe}$ concentrated over the radial range $3 \mathrm{kpc}<\mathrm{R}<12 \mathrm{kpc}$, a shallower abundance gradient for the older $\mathrm{PNe}$, which is in better agreement with our results.

Our previous results based on open clusters also show a shallower gradient for older clusters, but the results are quite uncertain since the cluster samples are collected from various literatures. Therefore, a uniform clusters sample is necessary in order to further explore the behavior of radial gradient and radial migration theory. This calls for a homogeneous spectroscopic survey of stellar clusters.

\section{LAMOST disk survey based on open clusters}

Open clusters have long been used to trace the structure and evolution of the Galactic disk. Since open clusters possess of relatively large age span whilst the cluster ages can be relatively accurately dated and one can see them to a large distance, their $[\mathrm{Fe} / \mathrm{H}]$ values serve as an excellent tracer of the abundance gradient along the Galactic disk as well as many other important disk properties, such as the age-metallicity relation (AMR), abundance gradient evolution, disk age, and so on (Chen et al. 2003).

Chen et al. (2003) has compiled an open cluster sample with metallicity, age, and distance data, as well as kinematic information available, from which an iron radial gradient of about $-0.063 \pm 0.008 \mathrm{dex} \mathrm{kpc}^{-1}$ is derived. This is quite consistent with the determinations of the oxygen gradient in nebulae and young stars. By dividing clusters into age groups, Chen et al. (2003) showed that the iron gradient was steeper in the past, which was confirmed by the results from Galactic planetary nebulae (Maciel et al. 2006). Both results support the inside-out Galactic disk formation mechanism, in which the invoked star formation rate and infall timescale vary with radius (Fu et al. 2009). 


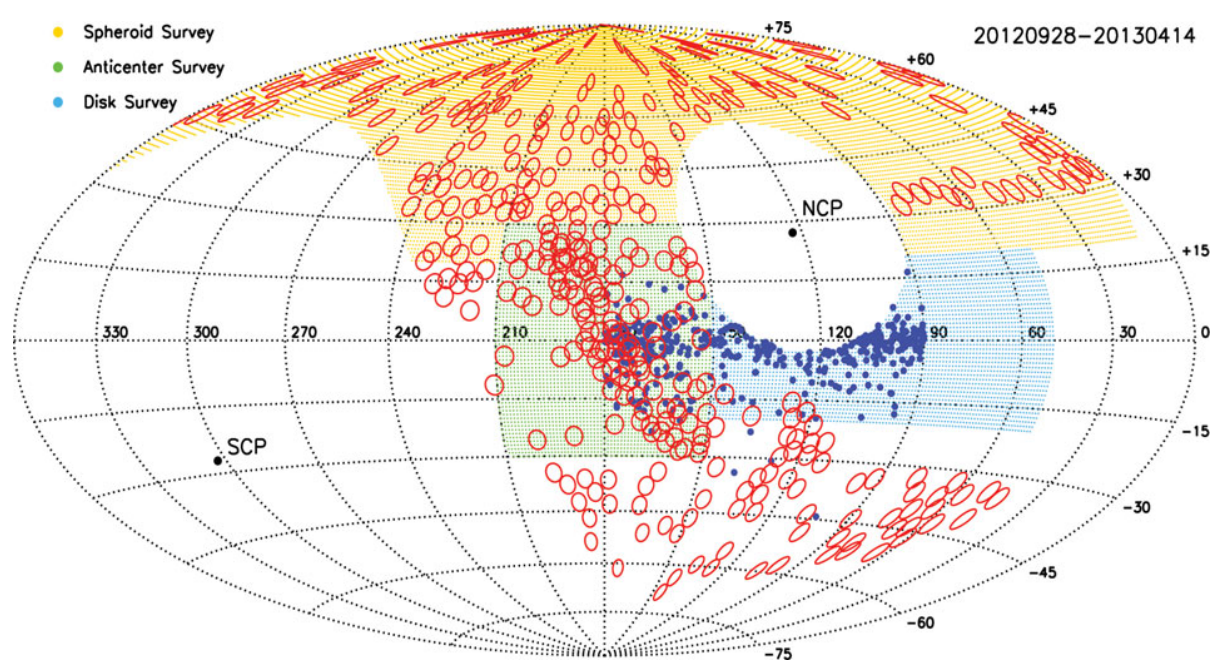

Figure 2. The positions of the disk open clusters in the Galactic Latitude Coordinates system (filled blue dots). Those positions will be covered by LAMOST low galactic survey in the following five years. The red circles are all the plates that have been observed during the past 6 months (from late September 2012 to April 2013). Shaded areas are the whole sky that LAMOST will survey during the five years.

However, the above results from open clusters are quite uncertain since the sample was derived from different sources. Based on the LAMOST (Deng et al. 2012; Chen et al. 2012; Liu et al. 2013, this meeting), we have proposed a low Galactic latitude disk survey concentrating on open clusters. One of the primary science objectives of the LAMOST disk survey is to investigate the structure of the thin/thick disks of the Galaxy, including the chemical abundance as a function of position within the disk and the extinction in the disk. In the main survey, we expect to cover 400 open clusters in the low Galactic latitude region, and obtain stellar radial velocities as well as abundance information for stars as faint as $r=16^{m}$ in the cluster fields. This will be the largest spectroscopic data sets for studying the properties of Galactic open clusters, as well as the structure, dynamics, and evolution of the disk as probed by open clusters.

In Figure 2, we shown the areas that the LAMOST survey will cover in the five years of observation (shaded areas). The red circles are those plates that LAMOST have observed between September 2012 and April 2013. The filled blue dots are the positions of open clusters that LAMOST can observe in Xinglong stations after considering the site conditions in LAMOST site (Yao et al. 2012).

Some of the expected scientific outputs from the disk open clusters survey include:

(a) significant improvements in essential parameter measurement of the targeted open clusters, using kinematic membership information and homogeneous abundance data.

(b) a database of spectroscopically confirmed cluster members in the outer parts of the clusters, that will provide good targets for detecting or verifying possible tidal tails of these stellar clusters.

(c) a large sample of young stellar objects across the surveyed area, which will provide important clues to studies of large-scale star formation and the history of Galactic star formation as well as information of the 3D extinction in the Galactic plane.

$(d)$ a more complete survey for the open clusters in the solar neighborhood, exploring the so-called abundance breaks around the solar radius. 
The open clusters are also particularly important to the survey project, since some of the well characterized open clusters provide important "standards" for a range of stellar types that can be used to calibrate the LAMOST observations and data-processing pipeline.

This work was supported by the NSFC grants 11173044 (PI Hou), 11073038 (PI Chen), Key Project 10833005 (PI Hou)

\section{References}

Andrievsky, S. M., Luck R. E., Martin P., Lépine J. R. D. 2004, A\&AA, 413, 159

Carraro, G., Ng, Y. K. \& Portinari, L. 1998, MNRAS, 296, 1045

Chen, L., Hou, J. L., \& Wang, J. J. 2003, AJ, 125, 1397

Chen, L., Hou, J. L. \& Zhao, J. L. 2008, IAUS, 248, 433

Chen, L., Hou, J. L., Yu, J. C., Liu, C., Deng, L. C. et al. 2012, RAA, 12, 805

Deng, L. C., Newberg, H. J., Liu, C. et al. 2012, RAA, 12, 735

Coşkunoğlu, B., Ak, S., Bilir, S. et al. 2012, MNRAS, 419, 2844

Friel E. D. 1999, ApSS, 265, 271

Fu, J., Hou, J. L., Yin, J. \& Chang, R. X. 2009, ApJ, 696, 668

Haywood, M. 2008, MNRAS, 388, 1175

Holmberg, J., Nordström, B. \& Andersen, J. 2009, A\&A, 501, 941

Hou, J. L., Prantzos, N., \& Boissier, S. 2000, A\& A, 362, 921

Lépine, J. R. D., Cruz, P., Scarano Jr., S. et al. 2011, MNRAS, 417, 698

Maciel, W. J., Lago L. G., Costa R. D .D. 2006, $A \mathscr{E} A, 453,587$

Magrini, L., Sestito, P., Randich, S., Galli, D. 2009, A\&SA, 494, 95

Nordström, B., Mayor, M., Andersen, J. et al. 2004, A\&A A, 418, 989

Pedicelli, S., Bono, G., Lemasle, B. et al. 2009, A\&A, 504, 81

Rudolph, A. L., Fich M., Bell, G. R., et al. 2006, ApJS, 162, 346

Scarano Jr., S. \& Lepine, J. R. D. 2013, MNRAS, 428, 625

Sellwood, J. A. \& Binney, J. J. 2002, MNRAS, 336, 785

Simpson, J. P., Colgan, S. W. J., Rubin, R. H. et al. 1995, ApJ, 444, 721

Stanghellini, L. \& Haywood, M. 2010, ApJ, 714, 1096

Steinmetz, M., Zwitter, T., Siebert, A., et al. 2006, AJ, 132, 1645

Twarog, B. A., Ashman, K. M., \& Anthony-Twarog B. J. 1997, AJ, 114, 2556

Wyse, R. \& Silk, J. 1989, ApJ, 339, 700

Yao, S., Liu, C., Zhang, H. T., et al. 2012, RAA, 12, 772

Yu, J. C., Sellwood, J. A., Pryor, C., Chen, L., \& Hou, J. L. 2012, ApJ, 754, 124

\section{Discussion}

JAQUES LÉPINE: You do not consider the hypothesis of metallicity breaks being a consequence of the corotation resonance?

Jinliang Hou: Yes, I do not mention this. The breaks could be related to the corotation resonance as you talked about in your presentation this morning. This need to be confirmed more precisely since for stars the distances are very crucial for the position of breaks. Open clusters are more reliable tracers for the breaks. 